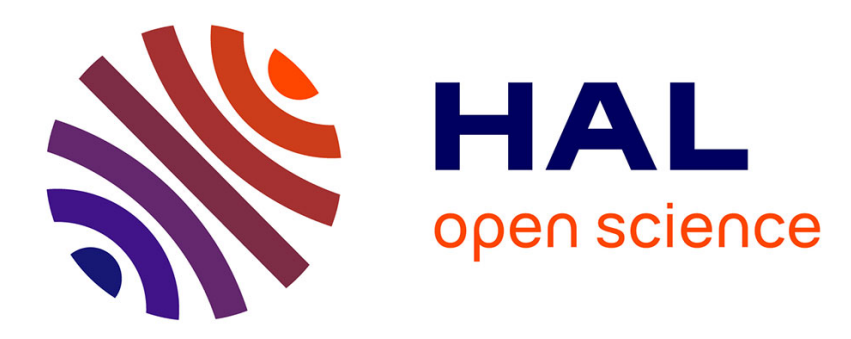

\title{
The electronic structure of the $\operatorname{InP}(110)$ surface
}

Y. Khazmi, P. Nilsson, J. Kanski

\section{To cite this version:}

Y. Khazmi, P. Nilsson, J. Kanski. The electronic structure of the $\operatorname{InP}(110)$ surface. Journal de Physique IV Proceedings, 1994, 04 (C9), pp.C9-199-C9-202. 10.1051/jp4:1994935 . jpa-00253496

\section{HAL Id: jpa-00253496 https://hal.science/jpa-00253496}

Submitted on 1 Jan 1994

HAL is a multi-disciplinary open access archive for the deposit and dissemination of scientific research documents, whether they are published or not. The documents may come from teaching and research institutions in France or abroad, or from public or private research centers.
L'archive ouverte pluridisciplinaire HAL, est destinée au dépôt et à la diffusion de documents scientifiques de niveau recherche, publiés ou non, émanant des établissements d'enseignement et de recherche français ou étrangers, des laboratoires publics ou privés. 


\section{The electronic structure of the $\operatorname{InP}(110)$ surface}

Y. Khazmi, P.O. Nilsson and J. Kanski

Department of Physics, Chalmers University of Technology, 41296 Gothenburg, Sweden

\section{ABSTRACT.}

As part of an extensive project on the surface electronic structure of semiconductor compounds we report here on an experimental and theoretical study of the $\operatorname{InP}(110)$ surface. By comparison of angle-resolved photoemission data with results from empirical tight-binding calculations it has been possible to identify electronic surface related features and specify their origin.

\section{INTRODUCTION.}

Many properties of semiconductor surfaces depend on the existence of electronic states localised at the surface. These states are conventionally classified as surface states (occurring in a band gap) or surface resonances (occurring in the continuum). Some specific surface states (resonances) on elemental and compound semiconductors have been identified and reproduced by calculations in literature. We have, however, not been able to find any case where the semiconductor surface has been fully characterised with respect to the electronic structure. By this we mean that the electronic states are not known over the whole valence region and over large parts of the Brillouin zone. Our aim is to carry out combined experimental and theoretical investigations to achieve this state. Our previous work has been focused on II-VI compounds. In the present report we carry over these investigations to III-V systems, exemplified here by InP. Some of the experimental data discussed here have been published in an earlier report [1].

\section{EXPERIMENTAL.}

Angle-resolved photoemission measurements were undertaken at the TGM beamline 41 of the national synchrotron radiation facility MAX in Lund, Sweden. The crystals were of $n$-type and obtained from MCP Electronic Materials Ltd. The (110) surface was prepared by cleaving in situ. The spectra were recorded with an angular and energy resolution of $2^{\circ}$ and $0.3 \mathrm{eV}$ respectively.

\section{THEORY.}

We represent the electronic structure by an empirical tight-binding LCAO scheme. Eight basis functions were used, i.e. the $s$ and $p$ states of the anion and cation atoms. First and second neighbour interactions 
(Hamiltonian matrix elements) were included, but the overlap integrals were set to zero [2]. A Green's function technique was applied to extract the local (partial) density of states in the different atomic layers [3]. The atomic positions in the first and second layers were taken from the recent calculation by Srivastava [4]. The matrix elements were modified in the surface relaxation region according to a simple quadratic scaling law [5].

\section{RESULTS AND DISCUSSION.}

Figure 1 displays photoemission energy distribution curves (EDCs) along the $\bar{\Gamma} \overline{\mathrm{X}}$ direction. All four borders of the surface Brillouin zone have been investigated but these results are reported elsewhere $[1,6]$. The bulk bands were established by an iterative fitting of the parametrised LCAO scheme to reproduce known energies, e.g. critical point energies observed in normal photoemission. The bulk derived features in the experimental spectra were identified by comparing with calculated direct transitions from the LCAO bands into free electron like states. The remaining features were considered as candidates for surface related states. The surface band structure $\mathrm{E}_{\mathrm{B}}\left(\mathrm{k}_{/ /}\right)$was directly extracted from the measured electron kinetic energy $\mathrm{E}_{\mathrm{kin}}$ and emission angle $\theta$ of the photoelectron:

$$
\begin{aligned}
& \mathrm{E}_{\mathrm{B}}=\mathrm{h} v-\mathrm{E}_{\mathrm{kin}}-\mathrm{e} \phi \\
& \mid \mathrm{k}_{/ /}=\sqrt{2 \mathrm{~m}} / \mathrm{h} \sqrt{\mathrm{E}_{\mathrm{kin}}} \sin \theta
\end{aligned}
$$
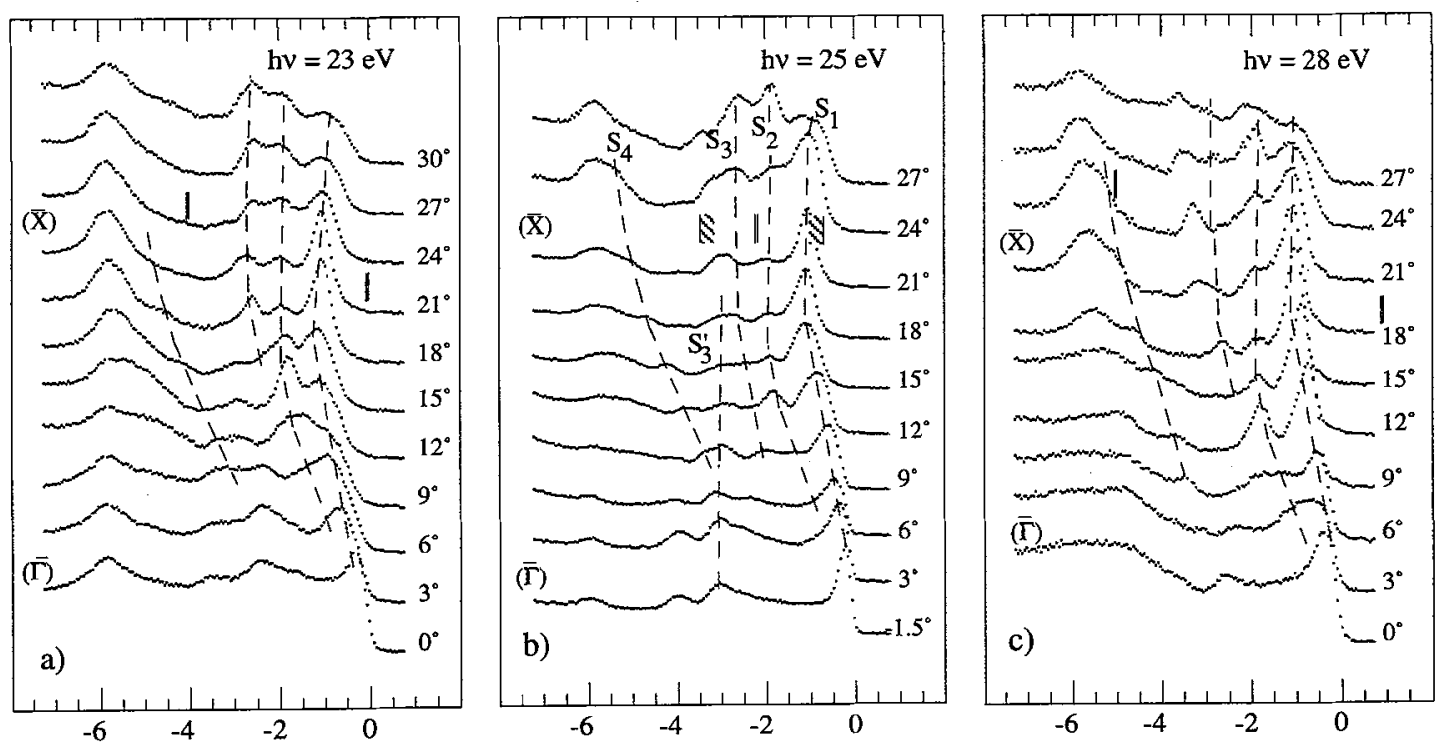

Binding energy rel. VBM (eV)

Figure 1. Valence band spectra from $\operatorname{InP}(110)$ along the $\bar{\Gamma} \bar{X}$ azimuth excited with 23,25 , and $28 \mathrm{eV}$ photons. In all cases the light was incident at $45^{\circ}$ and p-polarised in the (001) plane. The dashed lines indicate surface related structures as discussed in the text. The short vertical double-lines show the position of the $\bar{X}$ point. In the $21^{\circ}$ spectrum of the $25-\mathrm{eV}$ series we have aiso indicated the energy range around the $\bar{X}$ point defined by the angular resolution. 
Here $E_{B}$ is the binding energy, e $\phi$ the work function and $k_{/ /}$the crystal momentum parallel to the surface. Figure 2a summarises the peak locations in the $\bar{\Gamma} \bar{X}$ direction obtained in this way. Photon energies in the range $23-31 \mathrm{eV}$ were used. Since surface related states do not disperse with perpendicular momentum the $\mathrm{E}_{\mathrm{B}}\left(\mathrm{k}_{/ /}\right)$relation should not depend on the photon energy used (marked with different symbols). The lines are drawn as a guide for the eye. Figure $2 \mathrm{~b}$ shows our calculated peak positions of the local density of states in the first and second layer. The peaks can be identified either as surface states (resonances) or as (modified) bulk density of states.

A few photoemission reports on the InP(110) surface have appeared literature [7 - 9]. There also exist some theoretical papers on the surface band structure [10 - 12]. An overall, qualitative agreement is found between these reports. However the detailed agreement is rather poor, a fact which has motivated the present work.

Comparison between figures $2 \mathrm{a}$ and $2 \mathrm{~b}$ shows a good agreement and it is possible to identify the observed features. The upper state $S_{1}$ is the so called "dangling bond", which is a resonance in the $\bar{\Gamma} \bar{X}$ direction. It is entirely of $p$ character and is mostly located on the $P$ atom in the outermost atomic layer. The $S_{2}, S_{3}$, and $S_{3}$ ' states are of the same character, but with a small admixture from In p-states. $S_{2}$ has an appreciable
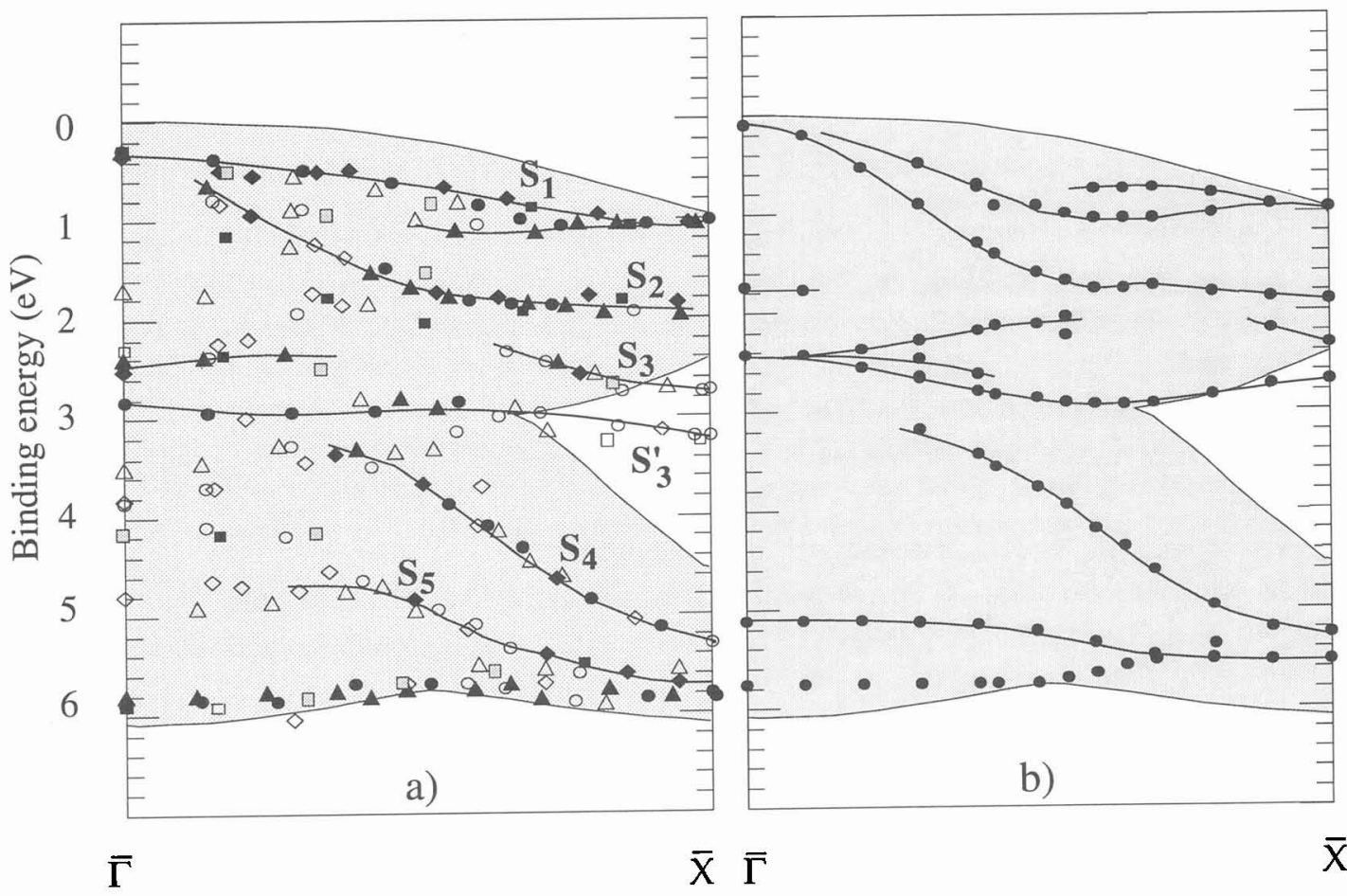

Figure 2a shows an experimental band structure plot of surface related structures for $23 \mathrm{eV}(\Delta), 25$ $\mathrm{eV}(\mathrm{o}), 28 \mathrm{eV}(\diamond)$, and $31 \mathrm{eV}(\square)$ photon energies. Filled symbols represent well defined peaks, open symbols weaker structures. The shadowed region is the surface projection of the bulk states. Figure $\mathbf{2 b}$ shows theoretical results for surface related structures. The points indicate peaks in the density of states in the two outermost layers and correspond to surface states (resonances) or surface modified bulk density of states. 
amplitude in the second layer, while $S_{3}$ and $S_{3}{ }^{\prime}$ in fact have their largest strength in the third layer. The $S_{4}$ peak is of a different nature. It has equal amounts of In $s$ and $P$ p character and it located entirely in the outermost layer. Its presence in the theoretical spectra depends on a reduced coupling between the first and second atomic layers. This coupling (In $\mathbf{s}$ and $\mathrm{P} s$ interaction) must be assumed to be considerably less than in the bulk. The $\mathrm{S}_{4}$ location and dispersion is not affected by the amount of reduction, only its intensity. In the same way we found $S_{5}$ to be located in the second layer as result of reduced ss $\sigma$ coupling between the second and third atomic layers. The structure indicated at $5-6 \mathrm{eV}$ binding energy (not joined by a line) is solely due to a density of states edge.

The other Brillouin zone borders are presently under investigation. From these results, together with further adjustments of the surface parameters, we expect to be able to establish the surface electronic structure of $\operatorname{InP}(110)$ in detail. Comparing with results on other compounds raises the interesting question if there is a "prototype surface band structure" for all the III-V and II-VI compounds, as in fact is the case for the bulk band structure.

\section{CONCLUSIONS.}

By comparing detailed angle-resolved photoelectron spectra from $\operatorname{In} P(110)$ with empirical tight-binding calculations it has been possible to identify observed surface related states. The restricted model used (minimal basis, second neighbour interactions, etc) seems adequate to describe the surface electronic structure.

\section{REFERENCES.}

[1] Qu H., Kanski J., Nilsson P. O., and Karlsson U. O., Surface Science 255 (1991) 237 - 244.

[2] See e.g. Schmeits M., Mazur A., and Pollmann J., Phys. Rev. B27 (1983) 5012 - 5031.

[3] See e.g. Lopez Sancho M.P., Lopez Sancho J.M., and Rubio J., J. Phys. F: Met. Phys. 15 (1985) 851 - 858.

[4] Srivastava G.P., Phys. Rev. B46 (1992) 7300 - 7303.

[5] Harrison W.A., Electronic Structure and the Properties of Solids (Freeman, San Francisco, 1980) p.149

[6] Khazmi Y., Nilsson P.O., and Kanski J., to be published

[7] McKinley A., Srivastava G.P., and Williams R.H., J. Phys. C13 (1980) 1581 - 1591.

[8] Williams G.P., Cerrina F., Lapeyre G.P., Anderson J.R., Smith R.J., and Hermanson J., Phys. Rev.B34 (1986) 5548 - 5557.

[9] Sorba L., Hinkel V., Middelmann H.U., and Horn K., Phys. Rev. B36 (1987) 8075 - 8081.

[10] Beres R.P., Allen R.E., and Dow J.D., Phys. Rev. B26 (1982) 5702 - 5705.

[11] Manghi F., Molinari, E., Bertoni C.M., and Calandra J., J. Phys. C15 (1982) 1099 - 1109.

[12] Mailhoit C., Duke C.B., and Chadi D.J., Phys. Rev. B31 (1985) 2213 - 2229. 